\title{
Un SOPLO DE VIDA EN LA PARED. ARTE RUPESTRE Y LA NOCIÓN DE PERSONA ENTRE los mayas del Posclásico de Laguna Mensabak, Chiapas
}

\section{A Breath of Life on the Wall. Rock Art and Personhood among the Post-classical Maya of Lake Mensabak, Chiapas}

\author{
Josuhé Lozada-Toledo* \\ Silvina Vigliani**
}

DOI: http://dx.doi.org/10.29043/liminar.v19il.798

\begin{abstract}
Resumen: El arte rupestre presente en los riscos de Laguna Mensabak expresa elementos vinculados a la noción y construcción de la persona maya durante el periodo Posclásico. Para ofrecer una aproximación a la manera en que se conceptualizó a la persona en el pasado, primeramente se hace una revisión de los conceptos nativos de persona mediante la información etnográfica y lingüística maya en el marco del modelo antropológico del personhood. Posteriormente, se analizan dos paneles rupestres del Risco Mensabak y se concluye con la propuesta que la noción maya de persona durante el Posclásico en la región era de carácter dividual y permeable.
\end{abstract}

Palabras clave: persona, entidad anímica, fuerza anímica, dividual, arte rupestre, mayas, Posclásico.

Abstract: The rock art found on the cliffs of Lake Mensabak evinces elements linked to the notion and construction of the Mayan person during the post-classical period. To approach the way in which the "person" was conceptualized in the past, we first review these native concepts through Maya ethnographic and linguistic information within the anthropological model of personhood. Subsequently, we analyze two rock art panels on the Mensabak cliff and conclude by proposing that the Maya notion of person during the post-classical period in the region had a dividual and permeable character.

Keywords: person, animistic entities, animistic force, rock art, Mayan Post-classic era.

\footnotetext{
* Josuhé Lozada Toledo. Doctor en Arqueología por la Escuela Nacional de Antropología e Historia, México. Profesor-investigador en la Dirección de Estudios Arqueológicos del Instituto Nacional de Antropología e Historia, Temas de especialización: arqueología maya, arqueología del paisaje, arte rupestre, Sistema de Información Geográfi ca. Correo electrónico: josuhe_lozada@inah.gob.mx. ORCID: https:// orcid.org/0000-0002-6203-2513

* Silvina Vigliani. Doctora en Arqueología por la Escuela Nacional de Antropología e Historia, México. Profesora-investigadora en el Insti-
}

tuto Nacional de Antropología e Historia, México. Temas de especialización: arqueología del paisaje, teoría de la agencia y personhood ontología relacional y animismo, arte rupestre y ritualidad prehispá nica. Correo electrónico: silvigliani@gmail.com. ORCID: https:/orcid. org/0000-0003-3818-6499

Enviado a dictamen: 6 de marzo de 2020.

Aprobación: 31 de agosto de 2020. 


\section{Introducción}

$\mathrm{U}$ no de los más grandes desafíos que presenta el estudio del pasado a través de la investigación arqueológica ha sido la identificación de las formas de ser y de conocer el mundo mediante bases ontológicas más cercanas a las de los grupos estudiados. Con base en ello, intentaremos rastrear y analizar elementos relativos a la noción y construcción de la persona maya a través de la información etnográfica y lingüística local, para luego incursionar en el análisis del arte rupestre presente en los riscos que bordean Laguna Mensabak, ubicada en la Selva Lacandona al noreste del estado de Chiapas.

Las principales interrogantes que plantea este estudio son: icómo los mayas posclásicos de Laguna Mensabak concibieron la noción de persona? y icómo transmitieron esta conceptualización a través del arte rupestre? Con la finalidad de proporcionar algunas posibles respuestas a estas preguntas, nuestro planteamiento retoma la antropología de personhood (Busby, 1997; Strathern, 1988) y sus aplicaciones en arqueología (Fowler, 2004; Gillespie, 2001; Jones, 2005, entre otros).

Este marco de referencia servirá para visualizar las diferentes tendencias de ser persona, particularmente distinguibles entre la noción de individuo, más propia de la sociedad occidental y moderna, y la noción dividual de persona, característica de sociedades no modernas. A su vez, nos permitirá entender características distintivas de lo dividual como son la permeabilidad y la partibilidad de la persona, en las que el flujo de sustancias y la circulación de objetos respectivamente representan aspectos significativos en la conformación de las personas humanas y no humanas en sus múltiples interacciones a lo largo de la vida y después de la muerte.

Finalmente, a partir del análisis del arte rupestre presente en el Risco Mensabak se dará cuenta de aquellos elementos iconográficos que dibujan el estado o condición de ser persona entre los mayas posclásicos, habitantes de la Selva Lacandona que plasmaron en las rocas su ser en el mundo.

\section{Las pinturas rupestres de Laguna Mensabak}

Laguna Mensabak se localiza al interior de la Selva Lacandona, en el noreste del estado de Chiapas (ver Figura 1). En medio de la espesa selva y bordeando la laguna emergen cinco riscos con presencia de arte rupestre, los cuales han sido nombrados por los lacandones como: Mensabak, Tsibaná, Kuh Nabaat, Jo’ton K'ak y Tanyok ${ }^{1}$ (ver Figura 2). La temporalidad asignada a la ocupación del área va desde el periodo Preclásico Medio (1000 a.C.-300 a.C.) hasta los tiempos históricos con una desocupación durante el periodo Clásico (300 d.C.-950 d.C.) (Lozada, 2017).

El estudio del arte rupestre de Laguna Mensabak comienza con algunas descripciones preliminares por parte de Cera (1977). Dicha información fue compilada posteriormente por Pincemin (1999), quien realizó un análisis comparativo entre estas pinturas y otras ubicadas en el estado de Chiapas. Estudios más sistemáticos comienzan con el trabajo de Sánchez (2005), quien además de describir los motivos rupestres aporta información acerca de los soportes rocosos donde están plasmadas las figuras. Posteriormente, Palka $^{2}$ (2005) vinculó el arte rupestre plasmado en los riscos con el paisaje sagrado, el cual analizó con ayuda de la información etnográfica recabada de los mayas lacandones, quienes aún habitan en la cercanía de dicha laguna.

Hacia el año $2012^{3}$ se el registro minucioso y total del arte rupestre de Laguna Mensabak, donde se dio a conocer la tipología de las pinturas rupestres y su conexión con el tema de las peregrinaciones en el paisaje de la Selva Lacandona. Actualmente, las pinturas rupestres de Laguna Mensabak han sido estudiadas bajo técnicas arqueométricas para conocer el tipo de minerales que usaban los antiguos mayas en la elaboración de sus pinturas rupestres. ${ }^{4}$

Para fines del presente trabajo nos centraremos en el Risco Mensabak por las temáticas identificadas en su arte rupestre. Se trata de un frente rocoso al borde del agua de cerca de 30 metros de altura con una extensión aproximada de 60 metros (Sánchez, 2005). Al risco con pinturas se accede exclusivamente en lancha o cayuco y 
sus grafismos hablan de posibles eventos ceremoniales con una naturaleza de carga ritual.

Las pinturas registradas en el Risco Mensabak suman un total de 123 y están distribuidas en siete paneles. En el Panel 1 se observan figuras antropomorfas, zoomorfas y algunos diseños abstractos, mientras que el Panel 2 presenta figuras antropomorfas con los brazos extendidos, manos al negativo, al positivo y manchas. El Panel 3 está conformado por figuras geométricas, zoomorfas, caritas y una figura de la deidad de la lluvia — dado que presenta anteojos, colmillos, sandalias y porta una bolsa de copal de la cual salen gotas de lluvia, similar a las imágenes posclásicas de dicha deidad que aparecen en el códice Borgia (Palka, 2005) - . El Panel 4 presenta manos al negativo, mientras que el Panel 5, dividido en dos sectores (el Sector Este y Sector Oeste) cuenta con manos al positivo. Por su parte, en el Panel 6, ubicado en el extremo oeste del Risco Mensabak, se observan dos figuras de animales, probablemente felinos, y dos manchas de pintura. Finalmente, el Panel 7 también se divide en dos sectores: el Sector Oeste con figuras abstractas, antropomorfas, zoomorfas, manos al negativo y manchas, y el Sector Este con figuras abstractas, zoomorfas, manos al positivo y manchas (Lozada, 2017).

Para este estudio analizaremos el Panel 1 y el Panel 5 del Risco Mensabak debido a su mejor estado de conservación y a los temas identificados en su arte rupestre (ver Figura 3). Ambos paneles se sitúan cronológicamente hacia el periodo Posclásico Tardío (1200 d.C.1550 d.C.), lo que coincide con una de las ocupaciones más significativas del área (Palka y Sánchez, 2011).

En la escena del Panel 1 (ver Figura 4), que puede ser descrita como de "estilo códice", 5 se observa un pequeño personaje, probablemente un niño, que está siendo presentado por dos adultos ante la deidad de la lluvia (Mensabak), la cual es posible identificar gracias a la presencia de dos figuras circulares que marcan los anteojos y algunos trazos verticales que se asemejan a los colmillos de la deidad. Una forma ondulada de color naranja sale de la boca del dios Mensabak, recorre la parte superior de la escena y pasa por la mano del pequeño personaje, para finalmente entrar y fusionarse por todo el cuerpo del niño. Los dos adultos también parecen alcanzados por esa forma ondulada de color naranja. En toda esta escena se observa que primero se pintaron los personajes en color rojo para posteriormente agregarse, en pintura naranja, la forma ondulada que alcanza al niño y toca a los personajes adultos, así como los ojos del dios Mensabak y una de las figuras zoomorfas de la parte superior del panel.

Los dos adultos ubicados a la derecha del niño aparecen sentados en posición ritual y presentan tocados en la cabeza, además de que uno de ellos porta una máscara. La presencia de máscaras refleja la relación que estos personajes tenían con el mundo de los dioses y su función de intermediarios con el mundo terrenal. La barba sujeta a la parte inferior de las máscaras indica que se trataba de una encarnación de personajes mayores, quizá ancestros (García, 2007).

Por su parte, el Panel 5 del Risco Mensabak (ver Figura 5) está conformado por dos grupos de improntas de mano en color rojo: un grupo compuesto de tres manos en el Sector Este y otro grupo de cinco manos en el Sector Oeste. A diferencia de las figuras pintadas, las improntas implican la participación directa de una parte del cuerpo de la persona, en este caso las manos. Existen varios ejemplos de manos pintadas en Mesoamérica, y en elárea maya los encontramos desde Chiapas (Lozada, 2011), Yucatán (Strecker, 1982), Guatemala (Woodfill y Henderson, 2016), hasta El Salvador (Lerma, 2014), entre otros.

A excepción del rostro, las manos constituyen una de las partes del cuerpo más representativas de la identidad de una persona, por lo que su impronta en la pared será única y diferente a las de otros sujetos. Tal vez, por ese aspecto identificatorio las manos sean uno de los motivos rupestres más repetidos a lo largo y ancho del planeta.

\section{La antropología de la persona: el modelo de personhood}

Partimos del supuesto de que el arte rupestre presente en los riscos de Laguna Mensabak expresa elementos vinculados a la noción y construcción de la persona maya. En tal 
sentido, hacemos un análisis de las pinturas y de la cultura material asociada a los riscos mencionados a partir de la antropología del personhood (Strathern, 1988), adoptada posteriormente por algunos arqueólogos (Fowler, 2004, 2008; Gillespie, 2001; Jones, 2005, entre otros).

La noción de personhood propone que la gente se constituye a partir de la totalidad de sus relaciones, tanto entre las diferentes personas como entre personas y objetos, lugares, rocas o animales (Fowler, 2004; Gillespie, 2001; Jones, 2005). La condición o estado de ser persona o personhood se define como un estado compuesto, que consiste de una variedad de aspectos como mente, alma, imagen, respiración, espíritu, sustancias, biografía, memoria, experiencia y nombre. Tales aspectos pueden residir en rasgos del cuerpo, pero también en otras cosas y lugares. Algunas cualidades pueden ser temporales, mientras que otras pueden ser eternas, ancestrales, o preexistir a la persona, e incluso prolongarse luego de su muerte (Fowler 2004).

La persona entonces aparece como una compleja amalgama de sustancias e influencias, en la que cada "ser" es una síntesis de la combinación de otros seres o sustancias que han contribuido social y físicamente para su existencia (Santos-Granero, 2009). A menudo, las discusiones sobre la condición de ser persona son expresadas fuera del contexto específico de las culturas, de sus propias concepciones y de sus prácticas. Sin embargo, este tipo de análisis requiere de un enfoque centrado en las formas en las cuales las personas son entendidas y construidas dentro de un contexto cultural particular (Kovasna, 2009).

Antes de dar paso a la identificación de los aspectos constitutivos de la persona maya, se delineará de manera general la concepción moderna de individuo, para luego analizar las tendencias dividuales de ser persona más comunes en sociedades no modernas. ${ }^{6}$

\section{Ser personas: lo individual y lo dividual}

Dentro de cualquier contexto en el que se desarrollan prácticas sociales existen tendencias específicas, creadas y reproducidas por las personas involucradas en tales prácticas y resultantes de procesos históricos particulares, que generan y reproducen diferentes modos de ser persona. En tal sentido, debe reconocerse que la noción de ser persona en Occidente -en especial en sectores urbanos y escolarizados- es una concepción originada en el humanismo filosófico, se cristaliza con el desarrollo de la modernidad, y está fundamentalmente caracterizada por la individualidad y la indivisibilidad, esto es, la noción de un individuo consciente de sí mismo, auto-contenido en el cuerpo y moralmente autónomo (Fowler, 2004; Thomas, 2004, 2007). Un rasgo prominente de la tradición intelectual occidental ha sido, a su vez, el de mantener a humanos y no-humanos separados al construir un conjunto interrelacionado de dicotomías que corren a lo largo de ejes de oposición dualista — sujeto/objeto, persona/ cosa, mente/cuerpo, humano/animal (Ingold, 2000)- . Estos dualismos, surgidos de la metafísica cartesiana, han tenido un potente impacto en la manera en que pensamos acerca de nosotros mismos en relación con animales, lugares, figuras y cosas no humanas.

En la práctica arqueológica se ha asumido implícitamente una conceptualización universal de la existencia humana, es decir, el hecho de que todos somos humanos y por lo tanto solo nos diferenciamos en la cultura que desarrollamos y cuyos productos materiales estudiamos. Sin embargo, la proyección acrítica de nuestra forma de ser en el mundo a tiempos y culturas distantes, como si aquella fuera una concepción universal, ha sido cuestionada al observarse que en sociedades tradicionales la gente asigna cualidades humanas - intelectuales, emocionales y subjetivas - a entidades no humanas, como animales, ríos, plantas, astros o rocas, con quienes además entablan vínculos sociales. Esto implica que la noción de persona abarca una amplia variedad de formas de las cuales el ser humano es solo una de ellas. ${ }^{7}$ Tales cuestionamientos dieron paso a los estudios de personhood y a la noción dividual de persona, definida inicialmente por Marilyn Strathern (1988) para las sociedades melanesias, y adoptada por otros investigadores en diferentes áreas (Bird-David, 1999; Busby, 1997; Fowler, 2004; Gillespie, 2001; Ingold, 2000; Jones, 2005; Santos-Granero, 2009; Vigliani, 2015, 2016; Willerslev, 2007). 
La dividualidad implica un estado de ser en el cual la persona es reconocida como compuesta y definida de manera múltiple. Esto significa que las personas se conforman a partir de las relaciones sociales que entablan con otras personas con quienes intercambian partes de sí mismos, ya sea objetivadas en elementos o a manera de sustancias. De este modo, algunos de los rasgos que componen a la persona dividual pueden estar fijos en la materia del cuerpo o pueden fluir a través de la persona. Todos los elementos del cosmos pueden pasar por la persona dividual afectando o modificando la constitución de esta. Dado que las relaciones están condensadas en sustancias físicas o en objetos que pueden ser intercambiados, así como en palabras o esencias espirituales, una persona dividual puede contener componentes de toda la comunidad dentro de sí misma (Fowler, 2004).

Fowler (2004) distingue dos formas de dividualidad: la partibilidad y la permeabilidad. La primera se expresa cuando la persona dividual es reconfigurada en la medida en que una parte de su ser es extraída (generalmente representada en un objeto o animal) y dada a otra persona en ciertas prácticas específicas. Así, por ejemplo, en los intercambios ceremoniales de Nueva Guinea los objetos regalados (por lo general, los cerdos al ser considerados entidades dividuales y de composición múltiple) son extraídos de la persona de la cual formaban parte, para entregarlos a otra persona y conformar así una relación. La parte extraída puede representar la totalidad de su ser, su esencia, su prestigio e incluso su personalidad. Partibilidad entonces significa que la persona que externaliza una parte de su ser a través de un regalo decrece en escala, mientras que aquello que da es absorbido por quien lo recibe. Luego, esa parte deberá ser devuelta de una forma diferente (Fowler, 2004).

La permeabilidad, por su parte, se expresa cuando la persona dividual es permeada por sustancias cuyas cualidades influyen en la composición interna de la persona. A diferencia de la anterior, las sustancias no son objetivadas y por lo tanto cambiantes, sino que son fijas, mientras que el cuerpo ya no es concebido como partible sino como un todo integral, pero con bordes permeables, de manera que las sustancias y la energía pueden fluir a través de ellos. En India, por ejemplo, cada sustancia tiene un género, un significado y un efecto que son fijos: tratar con sustancias calientes acentúa la masculinidad, mientras que tratar con sustancias frías acentúa la femineidad (Busby, 1997). En la Edad Media, el cuerpo contenía al alma y la mente, pero también era contenedor de varios humores. Estos fluían alrededor del cuerpo y se manifestaban en la flema, en la bilis o en la sangre. Cada humor afectaba el carácter de la persona de manera diferente: la gente sanguínea, por ejemplo, tenía un exceso de sangre, lo que la hacía más alegre que melancólica o malhumorada. Estas características podían modificarse al sangrarse o inducirse el vómito (Fowler, 2004).

De este modo, así como las personas pueden ser concebidas como seres individuales, conscientes de sí mismos y con límites corporales definidos, como en la moderna cultura occidental, también pueden ser reconocidas como seres múltiples formados a través de las relaciones con otros - humanos o no humanos-, estar constituidos de diferentes aspectos y extenderse por todo el mundo material, como suele ocurrir en muchas de las sociedades tradicionales que no han experimentado el proceso histórico de la modernidad (Vigliani, 2016). Cabe, al respecto, la síntesis que hace Bird-David al señalar que "cuando yo individualizo a un ser humano lo separo, pero cuando lo dividualizo, lo integro" (1999:72).

\section{La noción de persona entre los mayas}

La noción de la persona maya está íntimamente vinculada con la construcción del cuerpo. Al respecto, una obra fundamental y pionera en el estudio de la noción de persona en Mesoamérica es el libro Cuerpo humano e ideología. Las concepciones de los antiguos nahuas, de Alfredo López Austin (1984), texto obligado respecto al conocimiento de las entidades y fuerzas anímicas entre los grupos nahuas del centro de México. En dicha obra, López Austin demuestra la existencia de cierta unidad en los sistemas de pensamiento mesoamericano, lo que denomina como "núcleo duro", a partir de los datos históricos, etnográficos, lingüísticos e iconográficos. 
Para el área maya se cuenta con una vasta literatura etnográfica entre la que destacan los trabajos de Alfonso Villa Rojas (1987), María Dolores Cervera (2007) y Gabriel Luis Bourdin (2007), quienes estudiaron la construcción de la persona con mayas yucatecos, y el trabajo de Pedro Carrasco (1960), quien hizo lo correspondiente para chontales. En Guatemala, Barbara Tedlock (1982) analizó la corporalidad y principalmente el fenómeno del sueño entre los mayas quichés. De igual modo, los trabajos de Roberto Martínez González (2007, 2015) aportan datos sustanciales para el entendimiento de las entidades anímicas en el pensamiento maya.

Para el caso de Chiapas, existen trabajos con grupos tseltales y su noción del cuerpo por parte de autores como Pedro Pitarch $(1996,2000,2010)$ y Elios Figuerola Pojul (2000), así como los relativos a grupos tsotsiles en que destacan los trabajos clásicos de Calixta Guiteras Holmes (1965) y Evon Vogt (1966). Desde la perspectiva de la terapéutica indígena entre los tsotsiles y tseltales destacan los trabajos de Jaime Page Pliego (2002 y 2014), quien analiza la importancia del cuerpo en la noción de la persona indígena de Los Altos de Chiapas.

En los grupos tsotsiles de Los Altos de Chiapas la entidad anímica que ha sido más estudiada es el ch'ulel, que refiere a un alma inmortal e individual que puede perderse a causa del miedo, del susto o de una caída a la tierra o al agua, donde el ch'ulel se queda en el lugar del accidente (Guiteras, 1965).

También se cuenta con el wayjel, que refiere al alma animal que acompaña a la persona y es denominada entre los tseltales como lab (Pitarch, 2010). Se dice que la persona tsotsil completa cuenta con 13 ch'ulel y 13 wayjel (Page, 2001). Asimismo, se reconoce al ik' como aire que se manifiesta en dolores musculares (Guiteras, 1965) y al k'al o calor, que refiere a la energía vital de la persona tsotsil. De hecho, existe una correspondencia entre entidades y fuerzas anímicas, ya que en el ch'ulel reside la fuerza de la persona o el k'al (Page, 2001).

Entre los mayas lacandones de Chiapas se reconoce la entidad anímica denominada pixan, que designa una especie de cobertura corporal vinculada a la noción occidental de alma o espíritu (Boremanse, 1987). Para fines de este estudio, el pixan se utiliza como uno de los referentes etnográficos más cercanos por la ubicación de Laguna Mensabak en la Selva Lacandona, y dada la cercanía étnica y lingüística con los mayas yucatecos, hemos tomado la información relativa a las concepciones anímicas de ambos grupos.

Entre los mayas yucatecos existen dos entidades anímicas: ool y pixan y una fuerza anímica denominada $i k^{\prime}{ }^{8} \mathrm{El}$ ool refiere a la entidad anímica asociada al corazón; se dice que es la médula de la persona humana, e incluso es utilizado para referir al componente medular, tallo o cogollo tierno de los vegetales (Bourdin, 2007). El ool se define, asimismo, como la voluntad o capacidad de participación intencional, y refiere al mundo del sentido común, a las emociones, a la energía y al espíritu (Cervera, 2007). De acuerdo con López Austin (1984), esta categoría atribuida por los mayas yucatecos al corazón como centro principal de la vida anímica, la voluntad, el deseo, los sentimientos, los estados emotivos y las pasiones, se asemeja mucho a la categoría del tonalli desarrollado por los nahuas del centro de México (López, 1984). Esta similitud radica en que ambas entidades anímicas se desprenden del cuerpo durante el sueño, el coito, la ebriedad y, de modo accidental, a consecuencia de la caída y el susto (lo que puede provocar en estos casos la enfermedad y la muerte) (Martínez, 2007).

Los mayas yucatecos distinguen otra entidad anímica que denominan pixan. Esta expresión significa literalmente "envoltura" o "cobertura" de la persona. Se piensa que el pixan es el ánima de los difuntos que se separa del cuerpo con la muerte (Bourdin, 2007). El pixan de los mayas yucatecos en la actualidad se describe como un "gas caliente" que, al morir la persona, abandona el cuerpo disolviéndose en el aire (Bourdin, 2007). En el Diccionario Maya Cordemex (1980) también se define el pixan como "cosa que está cubierta"; asimismo, alude a lo dichoso, afortunado y bien aventurado. Otros autores señalan que el pixan hace referencia al alma de los muertos, se le recuerda y se le alimenta durante su retorno anual en el mes de noviembre, durante la fiesta de hanal pixan o "comida de las almas" (Ruz, 2003; Villa, 1987).

Es interesante mencionar que entre los mayas lacandones el pixan también hace referencia a una 
entidad anímica que se desprende del cuerpo tras la muerte para viajar sobre la laguna acompañada de un perro, y finalmente adentrarse en la roca en un área con presencia de pinturas rupestres. Cabe mencionar que el pixan de los lacandones no es exclusivo de los humanos; se cree que la selva, como lugar de vida e interacción, también está dotada en sí misma de un pixan (Balsanelli, 2018). Otra entidad anímica identificada para el caso de los mayas lacandones es el -or que refiere a los estados de ánimo o espíritu de una persona (características morales o anímicas del sujeto); además, se identifica al iḱ como una esencia que correspondería a una entidad gaseosa conectada con los procesos de respiración (Balsanelli, 2019).

Además del ool y el pixan, entre los mayas yucatecos se reporta la fuerza anímica denominada ik', y ella refiere al aliento, animación y vida. Se dice que se tiene $i k$ ' cuando se toma y se adquiere conciencia y responsabilidad. En ocasiones se manifiesta en las personas muertas como un remolino de espíritus (Cervera, 2007). De acuerdo con el Diccionario Maya Cordemex, el ik' entre los mayas yucatecos refiere al aire o viento, anhelito, resuello y soplo que uno echa por la boca (1980), por lo que el aliento no solo estaría ligado a la respiración, sino a la vitalidad, a la virtud y al poder. El aliento entonces refiere a algo más profundo que al acto mecánico de respirar (Martínez, 2015).

Si bien la literatura etnográfica es amplia respecto al tópico de la persona, no ocurre lo mismo para el caso de los mayas prehispánicos. Entre los pocos trabajos al respecto, destacan los de Houston, Stuart y Taube (2006), quienes retoman la metodología de López Austin (1984) para explicar la noción maya de corporalidad. Por su parte, y desde la epigrafía, Erik Velásquez García $(2009,2013)$ establece ciertas correspondencias con las entidades anímicas del periodo Clásico maya (Martínez, 2015). En este sentido, se ha identificado en la epigrafía maya el glifo way asociado a entidades compañeras, aunque con mayor seguridad refiere al sueño o acto del soñar (Houston y Stuart, 2001; Grube y Werner, 1994).

Por su parte, Gillespie (2001) analiza la noción de persona entre los mayas del periodo Clásico y la describe como un proceso constante que iba más allá de la vida de las personas. Centra su estudio en las inscripciones jeroglíficas y contextos funerarios de la élite, y advierte acerca de la importancia que tenía para la construcción de la persona maya no solo el nombre del gobernante, sino también el acto de nombrarlo, incluso después de la muerte (Gillespie, 2001). Asimismo, Landa sugiere que la práctica de depositar una cuenta de jadeíta en la boca del difunto probablemente refleje al ik' como una especie de aliento divino (Landa, 1994).

Si bien el ik' ha sido identificado como una especie de alma viviente o aliento sagrado en las inscripciones mayas del periodo Clásico, sus raíces se advierten hacia el horizonte olmeca o Preclásico, ya que si se comparan los signos de la escritura istmeña del Monumento E de Tres Zapotes, Veracruz (Velásquez, 2008) o del Altar 4 de Kaminaljuyú en Guatemala (Houston y Taube, 2000), por mencionar algunos ejemplos, es posible encontrar el signo de "viento" representado como una "T", y en el segundo caso, aparece además asociado con volutas de aliento que salen de un rostro antropomorfo.

En términos generales, el componente anímico que ha sido identificado con mayor frecuencia entre los mayas prehispánicos ha sido el ik', traducido como viento, aunque articula todo un conjunto de ideas sobre la vida. Así, el ik' está presente en las cosas que eran importantes para los mayas, como ciertas construcciones, lugares, objetos o animales, y requiere que sea implantado a través de un acto de creación (García, 2007).

Tanto las entidades como las fuerzas anímicas entre los mayas fueron importantes para definir a la persona y determinar su destino a través de la adquisición de responsabilidad. La responsabilidad jugó un papel fundamental en la construcción de la persona maya, especialmente en la transición de un estado infantil a un momento de madurez social.

Finalmente, como puede desprenderse de las referencias mencionadas, la información etnográfica ofrece una idea acerca de los modos de ser persona y la noción de corporalidad que se dieron en el pasado. Pensamos que el estudio del arte rupestre en el marco del modelo antropológico de personhood es una buena vía de análisis para indagar en tales temáticas. 


\section{La noción de persona en las pinturas rupestres de Laguna Mensabak}

\section{Panel 1 del Risco Mensabak}

Como fue descrito arriba, en la escena del Panel 1 de Laguna Mensabak se observa la figura de un niño que es presentado por dos adultos ante Mensabak, la deidad de la lluvia (ver Figura 4). Las características formales de los dos personajes (posición, presencia de tocados y máscara con barba) denotan la función que ellos tendrían como intermediarios entre las deidades y el mundo terrenal. Una forma ondulada de color naranja articula al niño, a la deidad y a los dos adultos en lo que parece ser una escena ritual.

Por lo anterior, es posible interpretar a los dos adultos como oficiantes o sacerdotes que presentan al niño ante el dios Mensabak para dotarle de la condición de persona o responsabilidad. En tal sentido, se trataría de un ritual de construcción de la persona del niño en el que el ik' o aliento vital, representado por la forma ondulada descrita arriba, sale de la boca del dios, se bifurca y, mediante la participación de los oficiantes, "permea" por completo al niño.

En un acercamiento a la imagen del niño (ver Figura 6), es posible observar que la figura ondulada no se dirige a su cabeza, sino a un área asociada al diafragma; por ello, es probable que se trate de un aliento o ik', más que de un ánima-corazón o pixan de los mayas lacandones (Balsanelli, 2019) u ool en términos de los mayas yucatecos. A su vez, el pequeño personaje parece sostener el elemento ondulado con una de sus manos, lo que insinúa que está controlando o recibiendo el aliento.

De este modo, esta fuerza anímica o $i k$ ', representada en este caso en la forma ondulada a la que nos referimos, siguió vigente entre los mayas posclásicos, articulando a la persona maya y sus deidades. De hecho, los dioses son considerados también personas o winik por los mayas, cuyo alimento es el copal y las esencias (Balsanelli, 2018).

Si, como dice Martínez González (2007), el aliento vital era insuflado por los dioses celestes al momento de la creación de la persona, dicho aliento o ik’ pudo ser, para los mayas posclásicos de Laguna Mensabak, la esencia o aliento del dios Mensabak fijada en los niños para obtener su condición de persona mediante el acto ritual.

En este proceso de reciprocidad ritual, bajo una especie de deuda, la persona maya tenía la responsabilidad de alimentar a las deidades. Al respecto, se han encontrado en las excavaciones realizadas al pie del Risco Mensabak evidencias de actividades rituales en la forma de ofrendas de comida y bebida, y probablemente de humo de copal y de sangre, dada la presencia de manos de metates y metates con restos de almidones (Cruz y Chaparro, 2018) asociados a la elaboración de salsas y atoles; así como de lítica tallada con restos de sangre (Pérez, 2019) y una capa de ceniza localizada justo debajo del risco (Lozada, 2019). Por otro lado, en los recorridos hechos por las paredes del Risco Mensabak se observan restos de manchas dejadas por posibles incensarios que luego cayeron hasta el pie de este. Actividades como estas pudieron responder a escenarios como el descrito para el Panel 1 del mismo risco, al tiempo que pudieron realizarse otras solicitudes ligadas a la atracción de las lluvias o al alejamiento de las tormentas.

En el centro de México existió un ritual similar al que proponemos para la escena del Panel l. Allí, los informantes de Sahagún (López, 1984) mencionan que a los niños se les ponía en contacto con el agua para pedir su ihiyotl a las deidades celestes. De acuerdo con Martínez González (2007), el ihiyotl de los nahuas estaba ligado al ecahuil (aliento de Ehecatl), y pensamos también que era un equivalente al ik' o aliento vital de los mayas.

Existen otros ejemplos etnográficos sobre rituales de construcción del niño como persona. Tal es el caso del meek' chähäl de los lacandones (Balsanelli, 2018) o el hetsmek' reportado entre los mayas yucatecos de Chankom, entre otras comunidades milperas (Redfield y Villa, 1964), y en Tusik, Quintana Roo (Villa, 1987), donde los padrinos del niño fungen también como mediadores para que el pequeño obtenga su condición de persona maya (Cervera, 2007).

Un detalle interesante es que debajo de la figura del niño hay una oquedad natural que, al parecer, fue apro- 
vechada por el pintor para resaltar la cueva, y con ello un lugar oscuro y frío asociado a los primeros años de vida de la persona maya. Al respecto, cuando un niño o niña nace es, para los mayas, un ser frío que paulatinamente aumenta de calor al llegar a la madurez. Si la relación entre la oquedad natural y la ubicación del Panel l fue intencional, podemos suponer que representa el paso de un estado frío del niño a otro más cálido en su proceso de construcción de persona.

Finalmente, si la interpretación de la escena es correcta, es plausible que las dos figuras zoomorfas del extremo superior derecho tengan una relación de consustancialidad entre el alter ego y el niño. Es decir, el componente anímico pudo estar asociado también a un animal, pues en diversos casos etnográficos, tanto del altiplano central mexicano (Romero, 2007) como del área maya (Pitarch, 1996), los animales suelen contribuir a determinar el carácter y el destino de la persona. Este animal externo comparte con el niño, probablemente, su mismo signo calendárico, personalidad, habilidades, virtudes, temperamento y destino (Villa, 1995). Dicho animal se asociaba con una fuerza calórica o k’ihn (vocablo en cholano Clásico) o k'inam (vocablo en maya yucateco). Ello nos inclina a pensar que se trata de un way o animal compañero o coesencia, ligado al onen ${ }^{9}$ de los lacandones.

\section{Panel 5 del Risco Mensabak}

El Panel 5 está ubicado en el Sector Este y Oeste del Risco Mensabak y consta de un conjunto de improntas de mano de color rojo (ver Figura 5). Al respecto, sabemos que en maya Clásico b'aah es imagen y 'ub'aah es "su imagen" o el "sí mismo" de la persona (Houston y Stuart 1998). También b'aah puede hacer referencia a figura, imagen pintada, reflejo y retrato, mientras que "ub'aah alude en ciertos contextos a "es su cuerpo" o "es su ser” (Velásquez, 2009:530). Si bien el b’aahis estaba primordialmente asociado a la cabeza, se extendió al cuerpo entero, a su imagen o reflejo y, por lo tanto, también a la mano.

En el Panel 5 las improntas de manos puedan estar vinculadas con la acción de implantar una parte de sí mismo en la roca. El "sí mismo" estaría entonces representado en el b'aah o "imagen", la cual simboliza o condensa en ella a la persona que la imprimió haciéndola presente en el Risco Mensabak.

\section{Comentarios finales}

Para el entendimiento de los mundos-de-la-vida de las sociedades pasadas es necesaria una revisión crítica de las categorías modernas que usualmente utilizamos los científicos sociales. Solo así es posible plantear hipótesis a partir de la analogía etnográfica, en tanto que es clave para lograr un acercamiento a las formas pasadas del personhood (Vigliani, 2015, 2016).

En un sentido analítico, los arqueólogos podemos considerar que las prácticas y principios asociados a la permeabilidad y la partibilidad pueden ser análogos a las practicadas por la gente en el pasado (Fowler, 2004). Ello abriría una gama de posibilidades sobre las distintas concepciones de ser persona en la antigüedad, cuando la cultura material fue central en la creación de su condición de persona y en la expresión de la agencia.

El acercamiento a la noción o estado de ser persona entre los mayas posclásicos es una tarea compleja, ya que no se cuenta con datos suficientes que proporcionen información sobre las entidades anímicas o fuerzas anímicas que caracterizaban a estos grupos. A su vez, debemos considerar que no hay un único modelo anímico compartido entre nahuas y mayas, por ejemplo (Martínez, 2006). Por el contrario, tanto sobre los grupos del centro de México como de Los Altos de Chiapas se ha registrado una amplia variedad de concepciones anímicas mesoamericanas con variantes regionales, locales e incluso personales (Martínez, 2015).

Dicho lo anterior, y a través de las evidencias presentadas en este trabajo, proponemos que la noción de persona entre los mayas posclásicos de Laguna Mensabak se construía sobre la base de una ontología relacional de carácter dividual y permeable. Si la interpretación del Panel l es correcta y la forma ondulada representa al aliento vital que fluye del dios Mensabak hacia el niño con la intermediación de dos oficiantes, 
estaríamos frente a un proceso de modificación de un estado de ser en otro, lo que podría corresponder a la construcción de la persona.

A su vez, en la concepción dividual de persona las sustancias con cualidades generativas pueden circular entre humanos, lugares, objetos, animales o dioses, por lo que el contacto con estas cosas tiene el potencial de modificar el estado o condición de la persona (Fowler, 2004, 2008). Esto nos conduce al Panel 5.

La imposición de las manos con pintura sobre los riscos con el fin de dejar una impronta puede tener muchas y diversas connotaciones. No obstante, cuando observamos en la literatura etnográfica que ciertos lugares conspicuos del paisaje como las lagunas, las cuevas y particularmente los riscos eran considerados como la personificación de los dioses (Palka y Sánchez, 2011; Lozada, 2017), es posible nuevamente que el carácter dividual y permeable de la persona se haga presente. En este caso, la acción de dejar la mano con pintura - y con ello del "sí mismo" - sobre la roca deificada implicaría algún tipo de relación que permee a la persona y a la comunidad toda con el dios local del fuego, la lluvia y la regeneración.

Finalmente, la posibilidad de acercarnos al estudio del arte rupestre y del paisaje desde un marco relacional y a través del modelo de personhood nos permite abordar su estudio de una manera más integral y ontológica, y culturalmente más cercana a la de los grupos que estudiamos. Ello nos invita a comprender mejor la participación de los riscos y sus pinturas en la vida social de los mayas posclásicos de Laguna Mensabak y captar el imperceptible instante de "un soplo de vida en la pared".

\section{Notas}

${ }^{1}$ Mensabak significa en maya lacandón "Señor que hace la pólvora o Señor de la lluvia”, Tsibaná "Señor de la escritura", Kuh Nabaat "Señor del Nabaat o esencia que se le echa al tabaco", Tanyok, refiere a otra deidad cuya traducción es problemática, pero está asociada a los pies.

${ }^{2}$ En el año 2005 comenzó el Proyecto Arqueológico Mensabak, dirigido por Joel W. Palka (Universidad Estatal de Arizona), quien con apoyo de su equipo de colabora- dores implementó una investigación arqueológica que hasta la fecha sigue vigente.

${ }^{3}$ El registro del arte rupestre de Laguna Mensabak se realizó desde el año 2012 mediante el proyecto arqueológico: "Registro de representaciones gráfico rupestres en torno a la laguna de Metzabok, Ocosingo, Chiapas", dirigido por Josuhé Lozada, desde El Colegio de la Frontera Sur (ECOSUR).

${ }^{4}$ Desde el año 2018 se cuenta con el proyecto denominado "Aplicación de técnicas arqueométricas en el estudio del arte rupestre de la Selva Lacandona", dirigido por Josuhé Lozada desde la Dirección de Estudios Arqueológicos del Instituto Nacional de Antropología e Historia (DEA-INAH).

${ }^{5}$ Se denomina "estilo códice" en el arte rupestre maya a aquel que por sus características formales se asemeja a la escritura tipo códice. Su temporalidad está asociada a contextos posclásicos (900 d.C.-1550 d.C.), aunque en la cerámica aparece desde el periodo Clásico Tardío (500 d.C. -850 d.C.). Se trata de un arte figurativo que se caracteriza por representaciones antropomorfas y zoomorfas estilizadas QUE en ocasiones va acompañado de deidades y símbolos calendáricos.

${ }^{6}$ No se trata de reificar aquí lo dividual ni de oponerlo a lo individual como dos formas de ser antagónicas y contradictorias, ya que elementos dividuales e individuales existen en todas las personas. Más bien, consideramos que tales conceptos pueden servir como herramientas interpretativas útiles a la hora de estudiar sistemas de relaciones.

${ }^{7}$ Existen numerosas etnografías que dan cuenta de ello. Entre estas destacan los trabajos de Descola (2001) entre los ashuar de la Amazonia ecuatoriana, de BirdDavis (1999) entre los nakaya del sur de la India, de Strathern (1988) entre los habitantes de las tierras altas de Nueva Guinea, de Viveiros de Castro (1996) entre los yawalapiti, entre muchos otros.

${ }^{8}$ La diferencia entre entidad anímica y fuerza anímica radica en que la primera refiere a una unidad estructurada con capacidad de independencia, en ciertas condiciones, del sitio orgánico en el que se ubica, (López, 1984); las entidades están asociadas a una parte del cuerpo (generalmente ubicadas en cabeza, corazón e 
hígado) y otras veces aparecen como una envoltura corporal; mientras que la fuerza anímica refiere a "aquellos elementos que, no siendo cuantificables ni individualizables, dotan de vida a la persona sin estar, por ello, directamente ligados a las funciones intelectuales. Una característica recurrente de las fuerzas anímicas es el estar vinculadas con fuentes externas que les permiten regenerarse y renovarse durante los ciclos ordinarios de la vida humana" (Martínez, 2007:154), como pueden ser el calor o la respiración.

${ }^{9}$ Entre los mayas lacandones, el parentesco se hace por vía paterna y se le conoce como onen, se trata de una afiliación de parentesco que corresponde a un animal. Los lacandones reconocen su pertenencia al determinado onen, e incluso piensan que cada persona tiene características físicas y conductuales del animal onen al que pertenecen (Vargas, 1998:63).

\section{Referencias}

Balsanelli, Alice (2019). “Almas lacandonas: estudio de las entidades anímicas de los Jach Winik”. En LiminaR. Estudios Sociales y Humanísticos, XVII(2), 115-130.

Balsanelli, Alice (2018). "Desde el punto de vista lacandón: propuesta para un nuevo acercamiento a la ontología lacandona”. En Cuicuilco. Revista de Ciencias Antropológicas, 72, 83-102.

Bird-David, Nurit (1999). "Personhood, Environment, and Relational Epistemology". En Current Anthropology, 40, 67-91.

Boremanse, Didier (1987). "La quête religieuse des Hach Winik (Indiens Lacandons) du Mexique meridional". En Journal de la Société des Américanistes, 73, 51-74.

Bourdin, Gabriel Luis (2007). "La noción de persona entre los mayas: una visión semántica”. En Revista Pueblos y Fronteras Digital, 2(4). Disponible en http:// www.pueblosyfronteras.unam.mx/index.php/index.php/pyf/article/view/218

Busby, Cecilia (1997). "Permeable and Partible Persons: A Comparative Analysis of Gender and the Body in South India and Melanesia". En Journal of the Royal Anthropological Institute 3(2), 261-278.
Carrasco, Pedro (1960). Pagan Rituals and Beliefs among the Chontal Indians of Oaxaca, Mexico. Berkeley, Los Ángeles: University of California Press.

Cera, Claire (1977). Peintures Rupestres Préhispaniques au Mexique. Mémoire de Maitrise, Universidad de Paris.

Cervera Montejano, María Dolores (2007). "El hetsmek' como expresión simbólica de la construcción de los niños mayas yucatecos como personas". En Revista Pueblos y Fronteras Digital, 2(4). Disponible en http:// http://www.pueblosyfronteras.unam.mx/index. php/index.php/pyf/article/view/224

Cruz, Jorge E. y Diana B. Chaparro (2018). Informe del análisis de almidones en piedras de molienda de Laguna Mensabak. México: Instituto de Investigaciones AntropológicasLaboratorio de Prehistoria y Evolución Humana-Universidad Nacional Autónoma de México.

Descola, Philippe (2001). "Construyendo naturalezas, ecología simbólica y práctica social”. En Philippe Descola y Gísli Pálsson (coords.), Naturalezay sociedad. Perspectivas antropológicas. México: Siglo XXI, pp. 101-123.

Diccionario Maya Cordemex (1980). Dirigido por Alfredo Barrera Vázquez. Mérida, Yucatán: Cordemex.

Figuerola Pujol, Helios (2000). "El cuerpo y sus entes en Cancuc, Chiapas". En Trace 38, pp. 13-24.

Fowler, Chris (2004). The Archaeology of Personhood. An Anthropological Approach. Londres y Nueva York: Routledge.

Fowler, Chris (2008). "Landscape and Personhood". En Bruno David y Julian Thomas (eds.) Handbook of Landscape Archaeology. California: Left Coast Press, pp. 291-299.

García, Valeria (2007). Imágenes materializadas: la máscara de jadeíta como retrato y representación en el Clásico maya. Tesis de maestría en Arqueología, INAH, ENAH, México.

Gillespie, Susan (2001). "Personhood, Agency, and Mortuary Ritual: A Case Study from the Ancient Maya". En Journal of Anthropological Archaeology, 20(1), 73-112.

Grube, Nikolai y Nahm Werner (1994). "A Census of Xibalba: A Complete Inventory of Way Characters on Maya Ceramics”. En J. Kerr (ed.), The Maya Vase Book, t. 4. Nueva York: Kerr Associates, pp. 686-715. 
Guiteras Holmes, Calixta (1965). Los peligros del alma. Visión del mundo de un tzotzil. México: Fondo de Cultura Económica.

Houston, Stephen D. y David S. Stuart (2001). "Peopling the Classic Maya Court". En T. Inomata y S. D. Houston (eds.), Royal Courts of the Ancient Maya, vol. 1: Theory, Comparison, and Synthesis. Boulder: Westview Press, pp. 54 - 83.

Houston, Stephen D. y David S. Stuart (1998). "The Ancient Maya Self: Personhood and Portraiture in the Classic Period". En Res. Anthropology and Aesthetics, 33, 73-101.

Houston, Stephen D., David S. Stuart y Karl A. Taube (2006). The Memory of Bones. Body, Being and Experience Among the Classic Maya. Austin: University of Texas Press.

Houston, Stephen y Karl A. Taube (2000). “An Archaeology of the Senses: Perception and Cultural Expression in Ancient Mesoamerica". En Cambridge Archaeological Journal, 10(2), 61-94.

Ingold, Timothy (2000). The Perception of the Environment. Essay on livelihood, dwelling and skill. Londres y Nueva York: Routledge.

Jones, Andy (2005). "Lives in fragments? Personhood and the European Neolithic". En Journal of Social Archaeology, 5(2), 193-224.

Kovasna, Anna (2009). Building Bodies, Balancing Powers of Insides, Outsides and Changing Notions of Male and Female Personhood Among the Matses of the Western Amazon. Tesis de maestría en Antropología, Universidad de Lund, Suecia.

Landa, fray Diego de (1994). Relación de las cosas de Yucatán. México: Consejo Nacional para la Cultura y las Artes.

Lerma Rodríguez, Félix (2014). Presencias en la roca. El arte rupestre de la gruta del Espíritu Santo. Tesis de doctorado en Historia del Arte, Universidad Nacional Autónoma de México, Ciudad de México.

López Austin, Alfredo (1984). Cuerpo humano e ideología. Las concepciones de los antiguos nahuas. México: UNAM, 2 t.

Lozada, Josuhé (2019). Informe de la temporada de campo 2018 del Proyecto Arqueológico: Aplicación de técnicas arqueométricas en el estudio del arte rupestre de la Selva Lacandona. In- forme entregado al Consejo de Arqueología de INAH, Ciudad de México.

Lozada, Josuhé (2017). El arte rupestre y la temporalidad del paisaje en Laguna Mensabak y Laguna Pethá, Chiapas. Tesis de doctorado en Arqueología, INAH, ENAH, Ciudad de México.

Lozada, Josuhé (2011). "Manos al negativo en cavidades zoques y mayas. Un acercamiento al lenguaje corporal". En Memorias del XXIV Simposio de Investigaciones arqueológicas en Guatemala, celebrado en julio de 2010. Guatemala: Museo Nacional de Arqueología y Etnología, pp. 1111-1123.

Martínez González, Roberto (2015). El nahualismo. México: Instituto de Investigaciones Históricas, Instituto de Investigaciones Antropológicas-UNAM.

Martínez González, Roberto (2007). "Las entidades anímicas en el pensamiento maya". En Estudios de Cultura Maya, XXX, 153-174.

Martínez González, Roberto (2006). "El ihiyotl, la sombra y las almas-aliento en Mesoamérica". En Cuicuilco. Revista de Ciencias Antropológicas, XIII(38), septiembre, 177-200.

Page Pliego, Jaime Tomás (2014). "La importancia del cuerpo en la noción de persona entre mayas actuales de Oxchuc, Chamula y Chenalhó, Chiapas". En Revista Pueblos y Fronteras Digital, 9(18), 35-48. Disponible en http://www.pueblosyfronteras.unam.mx/ index.php/index.php/pyf/\%20article/view/21

Page Pliego, Jaime Tomás (2002). Curandería tzotzil y procesos de formación, iniciación y de trabajo de sus practicantes. Tesis doctoral, Facultad de Filosofía y LetrasUNAM, México.

Palka, Joel (2005). "Rock Paintings and Lacandon Maya Sacred Landscapes". The PARI Journal. A Quarterly Publication of the Pre-Columbian Art Research Institute, 5(3), 1-7. Disponible en www.mesoweb.com/pari/publications/journal/503/PinturasRupestres.pdf

Palka, Joel y Fabiola Sánchez (2011). "Mujeres y hombres mayas posclásicos e históricos en la vida ritual en las tierras bajas de Chiapas". En Los investigadores de la cultura maya 2010, núm. 19, t. II. México: Universidad Autónoma de Campeche, 223-245. 
Pérez, Patricia (2019). Análisis funcional de artefactos líticos recuperados de las excavaciones arqueológicas en el Risco Mensabak. México: Escuela Nacional de Antropología e Historia.

Pincemin Deliberos, Sophia (1999). Manos y soles. Estudio de la gráfica rupestre en Chiapas. Tuxtla Gutiérrez: Gobierno del Estado de Chiapas, Universidad de Ciencias y Artes de Chiapas.

Pitarch Ramón, Pedro (2010). "Los dos cuerpos mayas. Esbozo de una antropología elemental indígena”. En Estudios de Cultura Maya, XXXVII, 149-178.

Pitarch Ramón, Pedro (2000). “Almas y cuerpo en una tradición tzeltal”. En Archives de Sciences Sociales des Religions, 112), 31-48.

Pitarch Ramón, Pedro (1996). Ch'ulel: una etnografía de las almas tzeltales. México: Fondo de Cultura Económica.

Redfield, Robert y Alfonso Villa Rojas (1964). Chan Kom. A Maya Village. Chicago, Londres: Chicago University Press.

Romero, Laura (2007). "La noción de persona y el concepto de ixtlamatki en la visión del mundo de los nahuas de la sierra negra de Puebla". En Revista Pueblos y Fronteras Digital, 2(4), 1-4l. Disponible en http://www.pueblosyfronteras.unam.mx/index. php/index.php/pyf/article/view/221

Ruz, Mario Humberto (2003). "Pasajes de muerte, paisajes de eternidad". En Alain Breton, Aurora Monod Becquelin y Mario Humberto Ruz (eds.). Espacios mayas: usos, representaciones, creencias. México: UNAM, Centro Francés de Estudios Mexicanos y Centroamericanos, pp. 619-657.

Sánchez Balderas, Fabiola (2005). "Arte rupestre en Metzabok. Una descripción preliminar”. En Revista Bolom, 2: 67-96. San Cristóbal de Las Casas, Chiapas: Centro de Investigaciones Frans Blom, Fundación Cultural Na Bolom A.C.

Santos-Granero, Fernando (2009). "Introduction: Amerindian Constructional Views of the World". En Fernando Santos-Granero (ed.), The Occult Life of Things. Native Amazonian Theories of Materiality and Personhood. Tucson: The University of Arizona Press, pp. 1-29.

Strathern, Marilyn (1988). The Gender of the Gift. Problems with Women and Problems with Society in Melane- sia. Berkeley, Los Ángeles, Londres: University of California Press.

Strecker, Matthias (1982) "Representaciones de manos y pies en el arte rupestre de cuevas de Oxkutzcab, Yucatán”. En Boletín de la Escuela de Ciencias Antropológicas de la Universidad de Yucatán, 9(52), 47-57.

Thomas, Julian (2004). Archaeology and Modernity. Londres y Nueva York: Routledge.

Thomas, Julian (2007). “Archaeology's Humanism and the Materiality of the Body". En Timothy Insoll (ed.), The Archaeology of Identities: a Reader. Londres y Nueva York: Routledge, pp. 211-224.

Tedlock, Barbara (1982). Time and the Highland Maya. Albuquerque: University of New Mexico Press.

Velásquez García, Erik (2013). "Nuevas ideas en torno a los espíritus wahyis pintados en las vasijas mayas: hechicería, enfermedades y banquetes oníricos en el arte prehispánico". En Estética del mal: conceptos y representaciones. México: Instituto de Investigaciones Estéticas-Universidad Nacional Autónoma de México, pp. 561-585.

Velásquez García, Erik (2008). "El desciframiento de la escritura istmeña: reevaluación del estado de la cuestión”. En Olmeca. Balance y perspectivas. Memorias de la Primera Mesa Redonda, t. II. Universidad Nacional Autónoma de México, Consejo Nacional para la Cultura y las Artes, Fundación Arqueológica Nuevo Mundo, pp. 639-665.

Velásquez García, Erik (2009). Los vasos de la entidad política de 'ik': Una aproximación histórica-artística. Estudio sobre las entidades anímicas y el lenguaje gestual y corporal en el arte maya Clásico. Tesis de doctorado en Historia del Arte, Facultad de Filosofía y LetrasUNAM, México.

Vigliani, Silvina (2016). "La noción de persona y la agencia de las cosas. Una mirada desde el arte rupestre". En Anales de Antropología, 50, 24-48.

Vigliani, Silvina (2015). Pinturas espirituales. Identidad y agencia en el paisaje relacional de sociedades cazadoras recolectorasy pescadoras. México: CONACULTA, INAH, ENAH.

Villa Rojas, Alfonso (1987). Los elegidos de Dios. Etnografía de los mayas de Quintana Roo. México: Instituto Nacional Indigenista. 
Viveiros de Castro, Eduardo (1996). "Os Pronomes Cosmológicos e o Perspectivismo Amerindio". En Mana, 2(2), 115-144.

Vogt, Evon Z. (1966). Los zinacantecos. Un pueblo tzotzil de los altos de Chiapas. México: Instituto Nacional Indigenista.

Willerslev, Rane (2007). Soul Hunters. Hunting, animism, and personhood among the siberian yukaghirs. Berkeley, Los Ángeles, Londres: University of California Press. Woodfill, Brent y Lucía R. Henderson (2016). "The Classic-Period Pictographs at Juliq' Cave, Alta Verapaz, Guatemala: an Interdisciplinary Approach to Cave Art as Organizing Principle". En Journal of Field Archaeology, 4l(2), $77-92$.

Figura 1. Mapa de ubicación de Laguna Mensabak

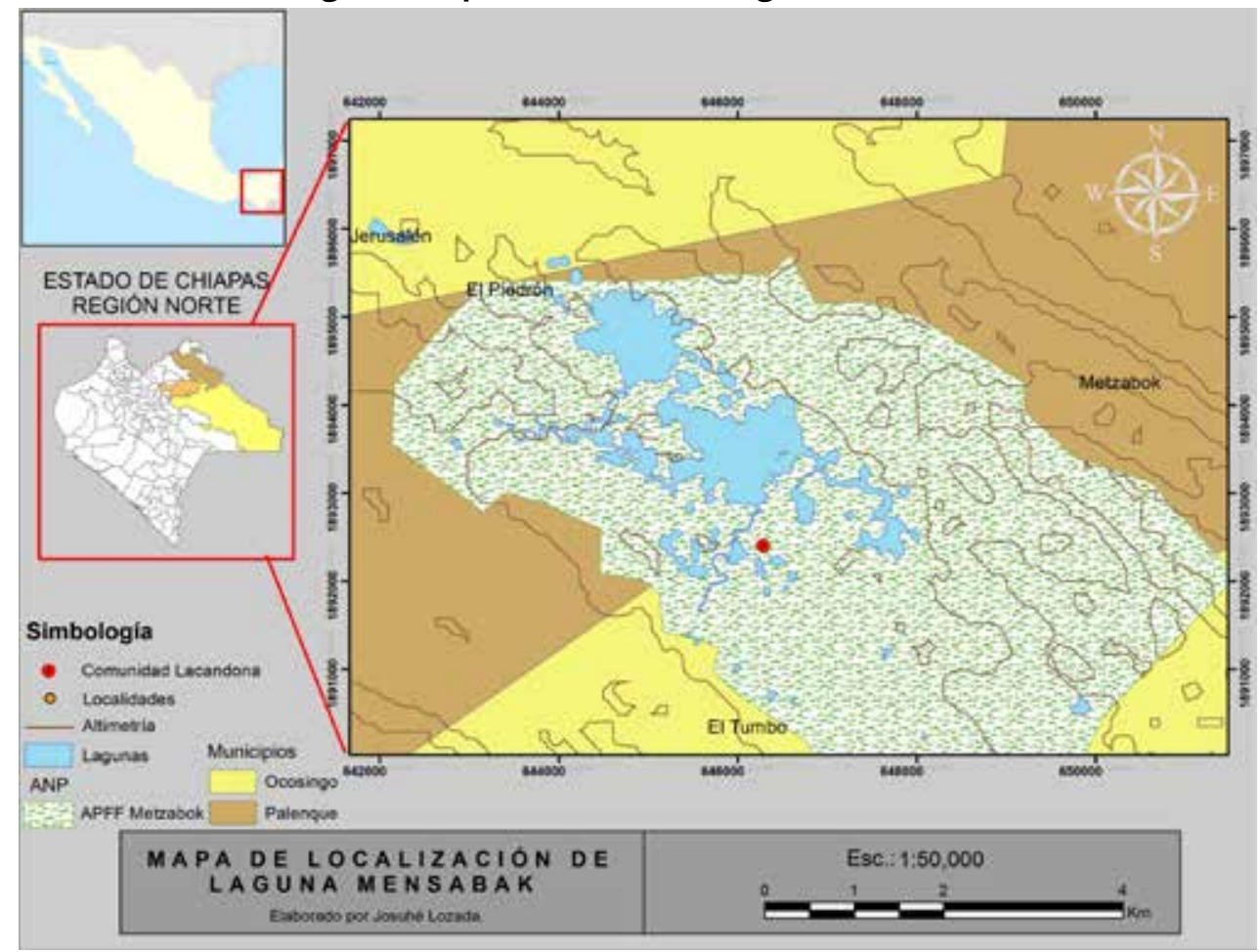

Fuente: Elaborado por Josuhé Lozada. 
Figura 2. Mapa de ubicación de los cinco riscos de Laguna Mensabak

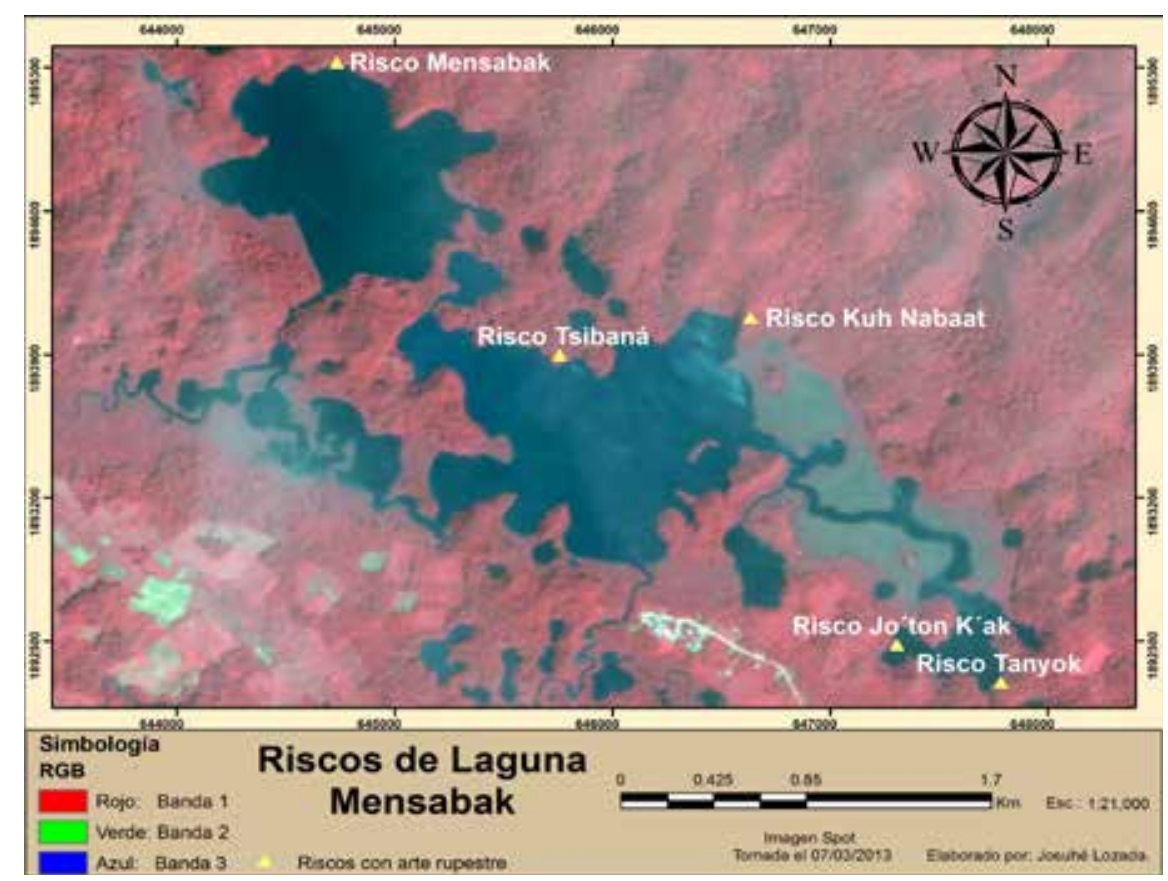

Fuente: Imagen Spot tomada el 07/03/2013. Elaborado por Josuhé Lozada.

Figura 3. Localización del Panel 1 y Panel 5 Sector Este y Oeste en el risco Mensabak

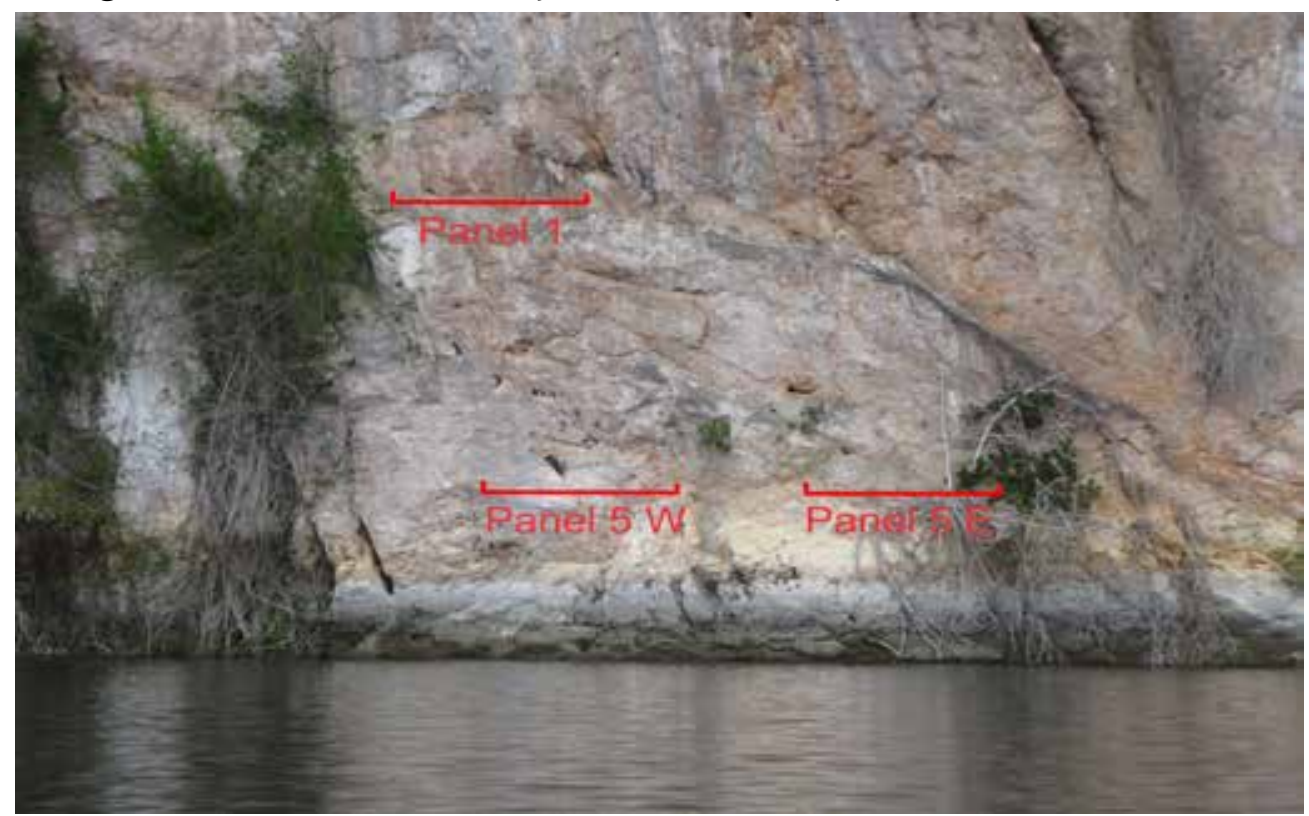

Fuente: Foto de Josué Gómez. 
Figura 4. Escena rupestre del Panel 1, Risco Mensabak

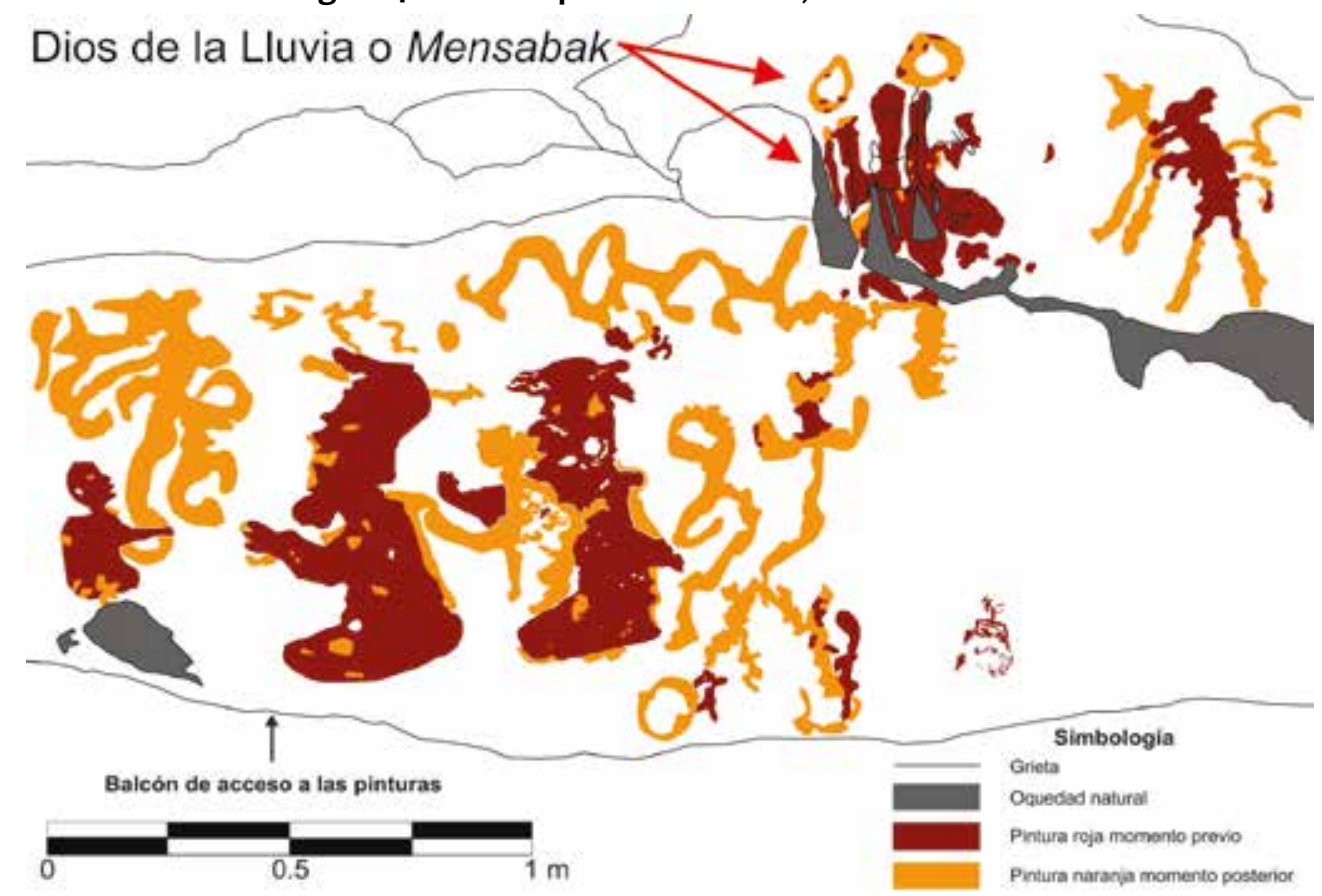

Fuente: Dibujo de Josuhé Lozada.

Figura 5. Dibujo final del Panel 5 Sector Este y Oeste del Risco Mensabak

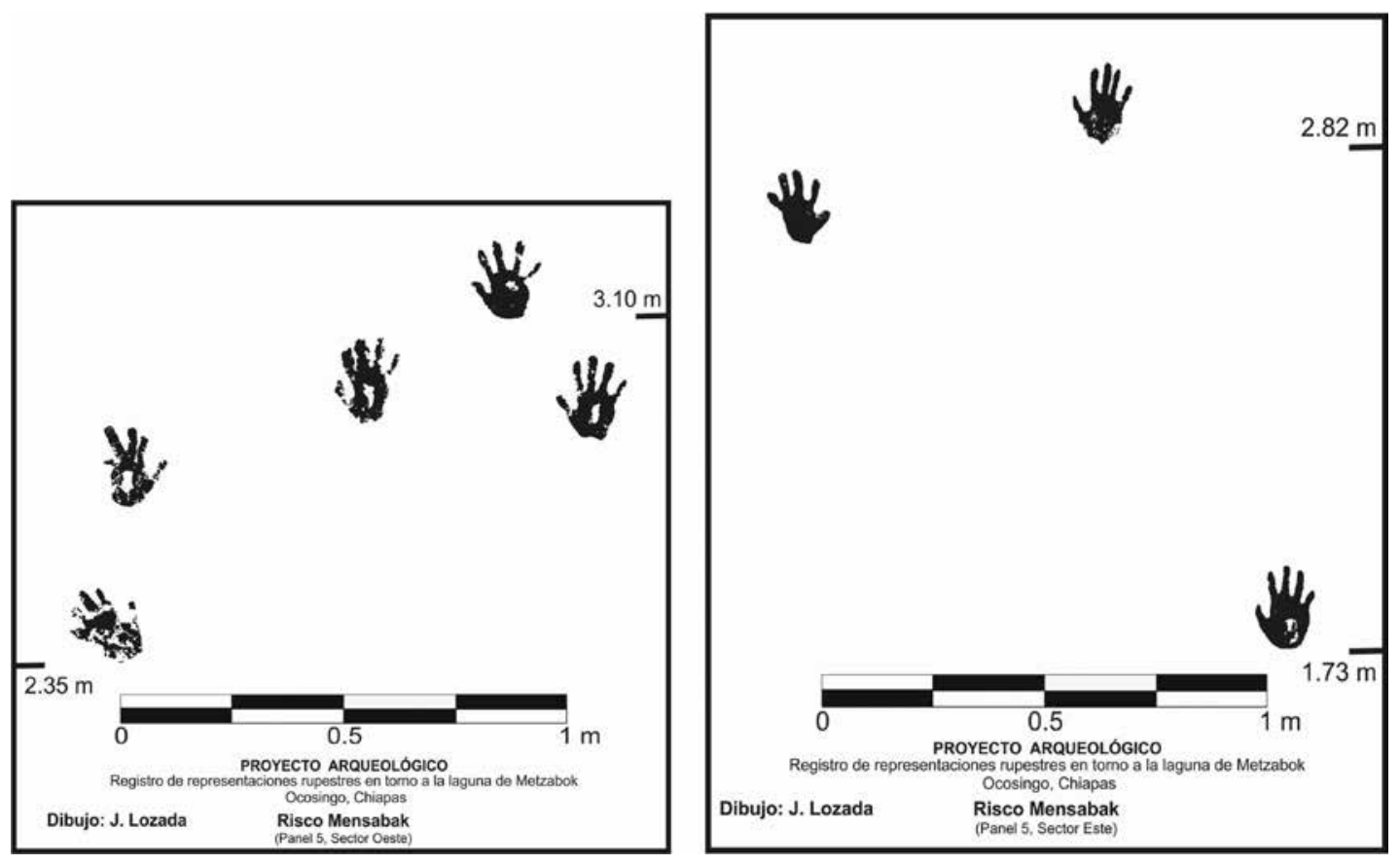

Fuente: Dibujo de Josuhé Lozada. 
Figura 6. Acercamiento al pequeño personaje de la escena rupestre del Panel 1 del Risco Mensabak

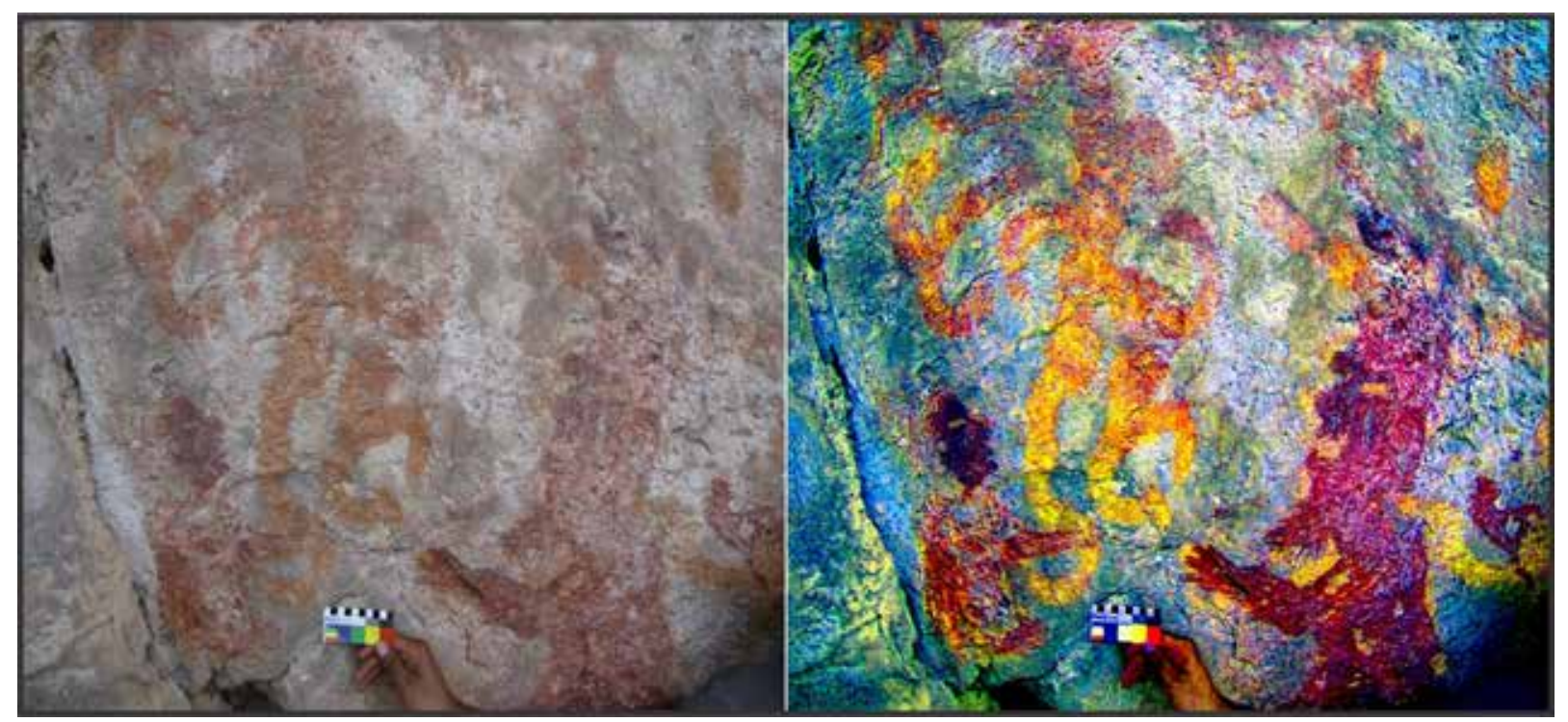

Fuente: Foto y procesamiento digital en DStretch por Josuhé Lozada, 2015. 